\title{
SHORT PROOF OF A THEOREM OF RADO ON GRAPHS
}

\section{B. L. FOSTER}

Berge's proof [1, p. 18] of Rado's theorem, a special case of the lemma in [2, p. 337], suffers from inaccuracies. In this note the result is derived from the following lemma of König $\left[1\right.$, p. 17]. If $\left(A_{1}, A_{2}, \cdots\right)$ is a sequence of nonempty, pairwise disjoint finite sets and $<$ is any relation between elements of consecutive sets such that for all $x_{n} \in A_{n}$, an element $x_{n-1} \in A_{n-1}$ exists with $x_{n-1}<x_{n}$, then a sequence $\left(a_{1}, a_{2}, \cdots\right)$ exists with $a_{n} \in A_{n}$, for all $n$, such that $a_{1}<a_{2}<\cdots$ $<a_{n}<\cdots$.

TheOREM (RADO). Given a locally finite graph, ${ }^{1}(G, \Gamma)$ a finite set of integers $K$ and a mapping $T$ of subsets of $K$ into subsets of $K$; if each finite subgraph, $\left(A, \Gamma_{\boldsymbol{A}}\right)$, admits a function $\phi_{\boldsymbol{A}}$ such that

$$
\phi_{A}(x) \in T\left\{\phi_{A}\left(\Gamma_{A} x\right)\right\}, \quad \text { for all } x \in A,
$$

then $(G, \Gamma)$ admits a function $\phi$ such that

$$
\phi(x) \in T\{\phi(\Gamma x)\}, \quad \text { for all } x \in G .
$$

Proof. Since there is no interaction between connected components, we may take $G$ to be connected (hence countable, because of local finiteness $\left[1\right.$, p. 18]). Let $G=\left\{x_{1}, x_{2}, \cdots\right\}$ and define a sequence of subsets of $G$ by setting $G_{n}=\left\{x_{1}, x_{2}, \cdots, x_{p_{n}}\right\}$, where $p_{1}$ is the least integer $r$ such that $r>1$ and $\left\{x_{1}, x_{2}, \ldots, x_{r}\right\}$ contains $\Gamma x_{1}$, and $p_{n}$, for $n>1$, is the least integer $r$ such that $r>p_{n-1}$ and $\left\{x_{1}, x_{2}, \cdots, x_{r}\right\}$ contains $\Gamma x_{1} \cup \Gamma x_{2} \cup \ldots \cup \Gamma x_{n}$. For each $n$, let $A_{n}$ be the set of all mappings $\phi: G_{n} \rightarrow K$ such that $\phi\left(x_{i}\right) \in T\left\{\phi\left(\Gamma x_{i}\right)\right\}$, for $i=1,2, \cdots, n$. Each $A_{n}$ is nonempty since it contains $\phi_{G_{n}}$, which exists by hypothesis. Moreover, each $A_{n}$ is finite and $A_{n}$ and $A_{m}$ are disjoint for $n \neq m$, since $G_{n} \neq G_{m}$. Define a relation between mappings in $A_{n-1}$ and $A_{n}$ by setting $\phi<\psi$ if and only if $\left.\psi\right|_{\theta_{n-1}}=\phi$, where $\left.\psi\right|_{G_{n-1}}$ is the restriction of $\psi$ to $G_{n-1}$. Then, for each $\psi \in A_{n}$, there is a $\phi \in A_{n-1}$ with $\phi<\psi$, in fact, $\phi=\left.\psi\right|_{a_{n-1}}$ will do. Thus, by König's result, there is a sequence $\phi_{1}<\phi_{2}<\cdots<\phi_{n}<\cdots$, with $\phi_{n} \in A_{n}$, for all $n$. Now define a $\phi$ on all of $G$ by $\phi\left(x_{n}\right)=\phi_{n}\left(x_{n}\right)$, for all $x_{n} \in G$. It is immediately verified that $\phi$ has the required property.

Received by the editors October 5, 1962 and, in revised form, July 3, 1963.

${ }^{1}$ A graph $(G, \Gamma)$, where $\Gamma$ maps elements of $G$ into subsets of $G$, is called locally finite if $\Gamma x$ and $\Gamma^{-1} x$ are finite, for every $x \in G$. 


\section{REFERENCES}

1. C. Berge, The theory of graphs and its applications, Wiley, New York, 1962.

2. R. Rado, Axiomatic treatment of rank in infinite sets, Canad. J. Math. 1 (1949), 337-343.

Marathon Oil Company, Littleton, Colorado

\section{CONCERNING CONTINUOUS IMAGES OF COMPACT ORDERED SPACES}

\section{B. TREYBIG ${ }^{1}$}

It is the purpose of this paper to prove that if each of $X$ and $Y$ is a compact Hausdorff space containing infinitely many points, and $X \times Y$ is the continuous image of a compact ordered space $L$, then both $X$ and $Y$ are metrizable. ${ }^{2}$ The preceding theorem is a generalization of a theorem [1] by Mardešić and Papić, who assume that $X, Y$, and $L$ are also connected. Young, in [3], shows that the Cartesian product of a "long" interval and a real interval is not the continuous image of any compact ordered space.

In this paper, the word compact is used in the "finite cover" sense. The phrase "ordered space" means a totally ordered topological space with the order topology. A subset $M$ of a topological space is said to be heriditarily separable provided each subset of $M$ is separable. If $a$ and $b$ are points of an ordered space $L$ and $a<b$, then $[a, b]$ $((a, b))$ will denote the set of all points $x$ of $L$ such that $a \leqq x \leqq b$ $(a<x<b)$, provided there is one; also, $[a, b]$ will be used even in the case where $a=b$. A subset $M$ of an ordered space $L$ is convex provided that if $a \in M, b \in M$, and $a<b$, then $[a, b] \subset M$. If $M$ is a subset of an ordered space $L$, then $G(M)$ will denote the set of all ordered pairs $(a, b)$ such that (1) $a \in M, b \in M$, and $a<b$, and (2) $\{a, b\}=M \cdot[a, b]$, provided there is one.

Lemma 0 . If $M$ is a compact subset of the ordered space $L$, then the relative topology of $L$ on $M$ is the same as the order topology on $M$.

Presented to the Society, May 4, 1962; received by the editors January 12, 1963 and, in revised form, July 3, 1963.

1 The author wishes to express his appreciation to the National Science Foundation for financial support.

${ }^{2}$ The referee has informed the author that the theorem of this paper was proved independently by A. J. Ward (Cambridge, England). 